Research Article

\title{
The Auxiliary Diagnosis and Imaging Characteristics of MRI Combined with CT in Patients with Cholangiocarcinoma
}

\author{
Jing Li $\left(\mathbb{O}^{1}{ }^{1}\right.$ Yuanlin Yu $\left(\mathbb{C}^{1},{ }^{1}\right.$ and Qing $\mathrm{He} \oplus^{2}$ \\ ${ }^{1}$ Department of Radiology, The First Affiliated Hospital of Fujian Medical University, Fuzhou, Fujian Province, China \\ ${ }^{2}$ Department of Radiotherapy, The First Affiliated Hospital of Fujian Medical University, Fuzhou, Fujian Province, China
}

Correspondence should be addressed to Qing He; xr212229@163.com

Received 18 June 2021; Revised 5 July 2021; Accepted 11 July 2021; Published 20 July 2021

Academic Editor: Muhammad Wasim Khan

Copyright (c) 2021 Jing Li et al. This is an open access article distributed under the Creative Commons Attribution License, which permits unrestricted use, distribution, and reproduction in any medium, provided the original work is properly cited.

Background. Patients with cholangiocarcinoma (CCA) have poor prognosis and high mortality. Therefore, early detection and early diagnosis are extremely important to control the development of CCA. This study aims to explore the diagnostic effect in patients with CCA and imaging characteristics of MRI combined with CT. Methods. 109 patients with suspected CCA underwent CT and MRI before diagnosis. The examination results were compared with the "gold standard." ROC curve was drawn to analyze the diagnostic efficacy of MRI combined with CT for CCA patients. Results. The diagnosis rate of suspected CCA patients was $95.41 \%$. The diagnostic coincidence rate of CT and MRI examination was $89.42 \%$ and $92.31 \%$, respectively. The diagnostic coincidence rate of MRI combined with CT examination was $100.00 \%$. The number of CT delayed enhancement, peripheral bile duct dilatation, and hepatic capsular depression were more than those of MRI. The number of circular enhancement cases in the CT group was less than that in the MRI group. ROC curve results showed that the sensitivity and specificity of MRI combined with CT for the diagnosis of CCA patients were higher than those of MRI or CT alone. Conclusion. MRI combined with CT has high diagnostic sensitivity and specificity and can provide imaging evidence for the clinical diagnosis and treatment of CCA.

\section{Introduction}

Cholangiocarcinoma (CCA) is a primary liver cancer that occurs in the bile ducts of the liver. The prognosis of CCA patients is poor and the mortality rate is high. Most patients cannot be cured, and a small number of patients with early CCA can be clinically cured. CCA lacks typical clinical symptoms in the early stage of onset. In the advanced stage of CCA, symptoms such as upper abdominal discomfort and hepatomegaly may appear [1]. Therefore, the clinical diagnosis and treatment of CAA is more difficult.

Previous studies have shown that CCA is a tumor that occurs from the confluence of the left and right hepatic ducts (hilar) to peripheral bile duct epithelial cells [2]. Histopathological examination is a commonly used diagnostic method for CCA patients and is regarded as the "gold standard." However, this method has poor reproducibility and requires the professional skills of doctors. It is easy to result in poor diagnosis compliance and tolerability in CCA patients [3, 4]. Therefore, simple and effective diagnostic methods are of great significance to patients with CCA.

CT is a commonly used imaging method, which has a good effect on the display of microcalcification in the lesion. MRI is more sensitive to intratumoral hemorrhage [5]. It has been shown that MRI combined with CT can give full play to the advantages of different imaging methods in the diagnosis and identification of malignant tumors [6]. At the same time, MRI combined with CT detection can evaluate the severity of the patient's disease according to different imaging features [7]. Therefore, this study aims to explore the auxiliary diagnosis in CCA patients and imaging characteristics of MRI combined with CT.

\section{Materials and Methods}

2.1. Patients. This is a retrospective study. 109 suspected CCA patients in the First Affiliated Hospital of Fujian Medical University were selected from February 2017 and 
December 2020. They are between 31 and 79 years old, with an average age of $59.39 \pm 5.65$ years old. The diameter of the tumor is between 23 and $135 \mathrm{~mm}$, with an average diameter of $64.29 \pm 6.61 \mathrm{~mm}$. The clinicopathological factors of these patients are shown in Table 1 . The study was approved by the Ethics Committee of the First Affiliated Hospital of Fujian Medical University. And the informed consent forms of these patients were obtained.

2.2. Inclusion and Exclusion Criteria. Inclusion criteria include the following: (1) patients are diagnosed by pathological examination and in line with CCA diagnostic criteria [8]. (2) Patients can tolerate and meet CT, MRI plain scan, or enhanced indications. (3) Patients have complete baseline and follow-up data.

Exclusion criteria include the following: (1) patients with mental disorders, cognitive dysfunction, or other malignant tumors; (2) patients receiving radiotherapy, chemotherapy, and biological immunotherapy before the examination; (3) patients with autoimmune system diseases and abnormal blood coagulation function.

2.3. Treatment Method. All patients were diagnosed by histopathological examination (gold standard). Before the diagnosis, all patients underwent CT and MRI examinations.

2.4. CT Examination. The Lightspeed VCT scanner is used to examine patients. Before the examination, the pathogenesis, clinical manifestations, and examination methods of CCA were explained to patients and their families to improve the cooperation of the patients. Patients were instructed to perform routine fasting examinations and routinely take $800-1000 \mathrm{~mL}$ of negative contrast agent before the examination. During the examination, the patient was placed in a supine position. And the relevant parameter settings were completed according to the patient's condition. Finally, the unscanned and enhanced $5 \mathrm{~mm}$ layer thickness image was reconstructed as a $0.625 \mathrm{~mm}$ image. And the coronal and sagittal image reconstruction was completed $[9,10]$.

2.5. MRI Examination. After completing the CT examination, MRI examination is performed according to the patient's wishes. The instrument is a $3.0 \mathrm{~T}$ Inegenia superconducting magnetic resonance scanner (Philips, Netherlands) using a 32-channel volume phased array coil. It takes about 18-23 s to complete the liver scan and imaging. Relevant parameters were set according to the patient's condition. The obtained images are transferred to the processing software. The examination results are compared with the "gold standard." Finally, the diagnostic coincidence rate of different imaging examinations is analyzed. And the imaging characteristics of CT and MRI are recorded $[11,12]$.

2.6. Statistical Analysis. SPSS24.0 software was used to analyze the data. The difference between the two groups was analyzed by the $\chi^{2}$ text. The ROC curve was used to analyze the diagnostic efficacy (sensitivity and specificity) of MRI combined with CT in CCA patients. $P<0.05$ indicates that the difference is statistically significant.

\section{Results}

3.1. Comparison of the Diagnostic Coincidence Rate of MRI and CT in Patients with CCA. Among the 109 suspected CCA patients, 104 patients were confirmed by histopathological examination. And the diagnosis rate was $95.41 \%$. CT examination confirmed 93 cases, and the diagnosis coincidence rate was $89.42 \%(P<0.05$, Figure 1$) .96$ cases were confirmed by MRI examination. The coincidence rate of MRI diagnosis was $92.31 \%(P<0.05$, Figure 1). 104 cases were diagnosed by MRI combined with CT examination, and the diagnosis coincidence rate was $100.00 \%(P>0.05$, Figure 1).

\subsection{Comparison of Imaging Characteristics between MRI and} CT in Patients with CCA. The CT group and the MRI group had no significant difference in enhancement, "fast-in and fast-out" enhancement, smooth enhancement ring, flatness, hepatic lobe atrophy, DWI high signal, and abnormal perfusion $(P>0.05$, Table 2$)$. The number of delayed enhancement, peripheral bile duct dilation, and hepatic capsule depression in the CT group were more than those of MRI $(P<0.05$, Table 2$)$. The number of uneven enhancement cases in the circular-enhanced CT group was less than that of the MRI group $(P<0.05$, Table 2$)$.

3.3. Diagnostic Efficacy of MRI Combined with CT in Patients with CCA. ROC curve results showed that the sensitivity and specificity of MRI combined with CT for CCA patients were higher than those of MRI and CT alone $(P<0.05$, Table 3, Figure 2).

\section{Discussion}

CCA is a malignant tumor with high clinical incidence. At present, it is generally believed that the incidence of CCA is related to intrahepatic bile duct stones, long-term chronic inflammation, or related physical and chemical stimulation $[13,14]$. Even worse, the clinical diagnosis and treatment of CCA is difficult because of the lack of typical clinical symptoms in the early stage of CCA. Therefore, most patients are already in the middle and advanced stages at the time of diagnosis. Now, histopathological examination is still the "gold standard" for the diagnosis of patients with CCA. Although pathological examination is helpful for diagnosis, diagnostic compliance and tolerance are poor $[15,16]$. Therefore, it is very important to explore new diagnostic methods of CCA in clinical practice.

In recent years, MRI combined with CT has been used in patients with CCA. Moreover, the diagnostic accuracy of MRI combined with CT is satisfactory [17]. In this study, 104 of 109 suspected CCA patients were confirmed by histopathological examination. The diagnosis rate of histopathological examination was $95.41 \%$. In addition, the diagnosis 
TABLE 1: Clinicopathological information of 109 patients with cholangiocarcinoma.

\begin{tabular}{lccccccccc}
\hline \multirow{2}{*}{ Features } & \multicolumn{2}{c}{ Gender } & \multicolumn{3}{c}{ Symptom } & \multicolumn{3}{c}{ Examination } \\
& Male & Female & Abdominal pain & Jaundice & Fever & Fatigue & CT & MRI & CT + MRI \\
\hline Cases & 65 & 44 & 32 & 16 & 19 & 29 & 57 & 29 & 23 \\
\hline
\end{tabular}

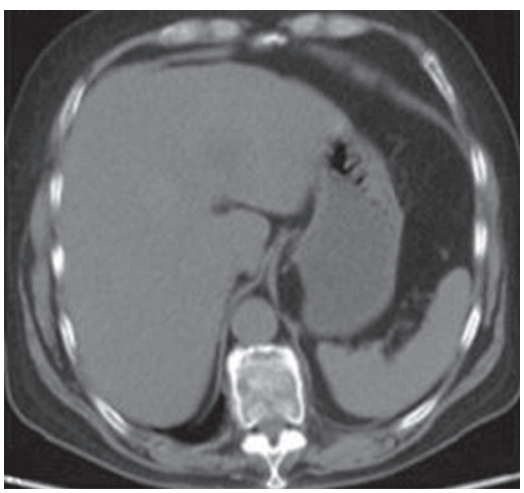

(a)

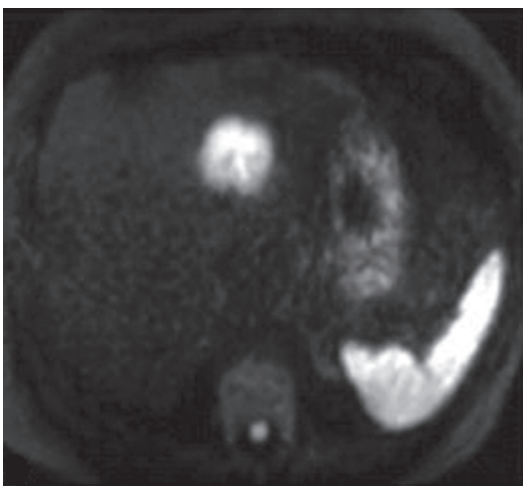

(d)

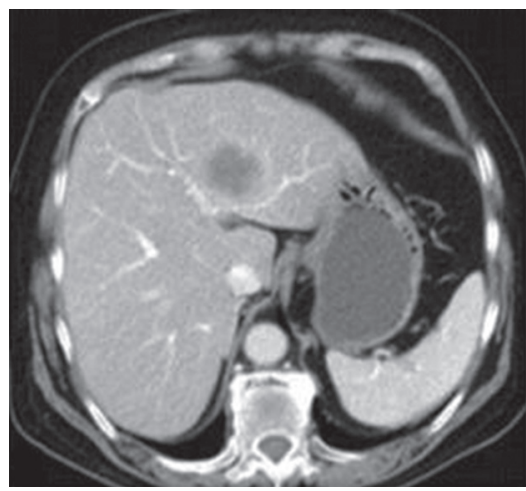

(b)

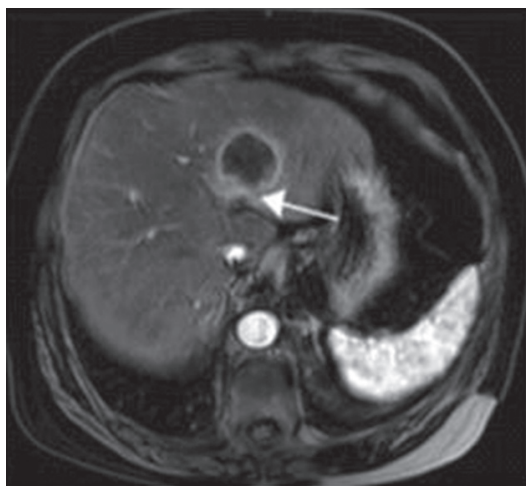

(e)

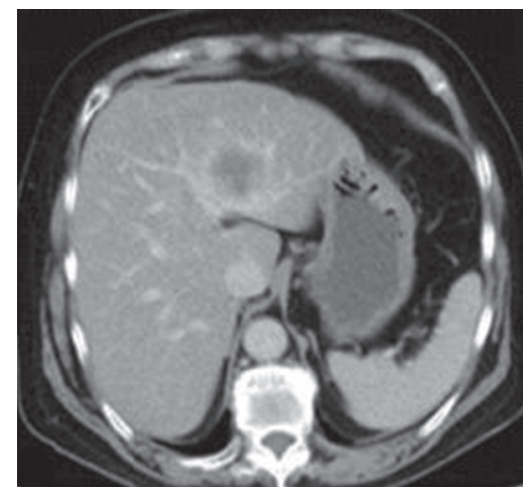

(c)

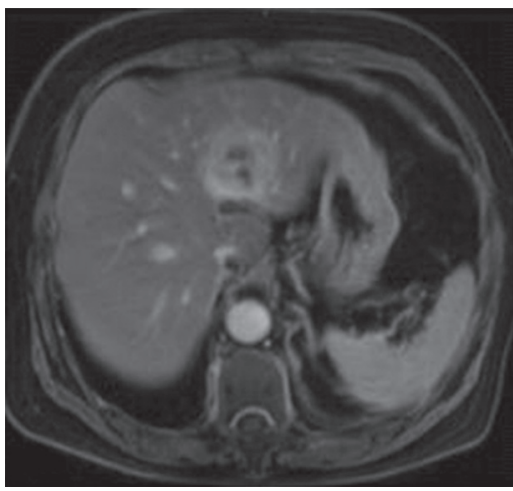

(f)

FIgURE 1: Typical pathological MRI and CT images. In 63 patients with CCA, the lesion was located in the left lobe of the liver. And all patients underwent MRI and CT examinations. (a) CT picture of a typical case, (b) a circular enhancement at the edge of the lesion in the arterial phase, (c) an enhanced inward filling in the equilibrium phase, and (d) DWI picture of the MRI shows high signal intensity. (e) A circular enhancement shows at the edge of the tumor, but the strengthening ring is incomplete. (f) The inward filling of the mass in the equilibrium phase.

TABLE 2: Comparison of imaging characteristics between MRI and CT in patients with CCA ( $n(\%))$.

\begin{tabular}{|c|c|c|c|c|c|}
\hline & Characteristics & $\mathrm{CT}(n=57)$ & MRI $(n=29)$ & $x^{2}$ & $P$ \\
\hline \multirow{6}{*}{ Enhanced features } & Annular strengthening uneven strengthening & $41(71.93)$ & $28(96.55)$ & 6.351 & $0.035^{*}$ \\
\hline & Delayed reinforcement & $14(24.56)$ & $3(10.34)$ & 9.672 & $0.021^{*}$ \\
\hline & No obvious enhancement & $2(3.51)$ & $0(0.00)$ & 1.491 & 0.958 \\
\hline & "Fast-in and fast-out" enhancement & $2(3.51)$ & $0(0.00)$ & 1.491 & 0.958 \\
\hline & Smooth and flat strengthening ring & $0(0.00)$ & $0(0.00)$ & 0.000 & 1.000 \\
\hline & DWI high signal & $57(100.00)$ & $29(100.00)$ & 0.000 & 1.000 \\
\hline \multirow{4}{*}{ Arterial passage lesions } & Peripheral bile duct dilation & $29(50.88)$ & $16(55.17)$ & 6.094 & $0.020^{*}$ \\
\hline & Depressed liver capsule sign & $42(73.68)$ & $11(37.93)$ & 7.103 & $0.013^{*}$ \\
\hline & Hepatic lobe atrophy & $9(15.79)$ & $5(17.24)$ & 1.593 & 0.615 \\
\hline & Abnormal perfusion & $18(31.58)$ & $10(34.48)$ & 0.834 & 0.058 \\
\hline
\end{tabular}

${ }^{*} P<0.05$.

coincidence rate of CT and MRI examination was $89.42 \%$ and $92.31 \%$, respectively. More importantly, the diagnosis coincidence rate of CT combined with MRI reached $100.00 \%$ in this study. These results indicate that the diagnostic accuracy of MRI combined with CT is very high. Qi et al. [18] also reported that MRI combined with CT can achieve a 
TABLE 3: Diagnostic efficacy of MRI combined with CT in patients with CCA.

\begin{tabular}{|c|c|c|c|c|c|c|c|}
\hline \multirow{2}{*}{ Variable } & \multirow{2}{*}{ AUC } & \multirow{2}{*}{ SD } & \multirow{2}{*}{$P$} & \multicolumn{2}{|c|}{$95 \%$ confidence interval } & \multirow{2}{*}{ Sensitivity } & \multirow{2}{*}{ Specificity } \\
\hline & & & & Lower limit & Upper limit & & \\
\hline $\mathrm{CT}$ & 0.746 & 0.061 & $P \leq 0.001$ & 0.713 & 0.858 & 0.794 & 0.671 \\
\hline MRI & 0.812 & 0.074 & $P \leq 0.001$ & 0.794 & 0.871 & 0.841 & 0.657 \\
\hline $\mathrm{CT}+\mathrm{MRI}$ & 0.894 & 0.083 & $P \leq 0.001$ & 0.845 & 0.932 & 0.931 & 0.612 \\
\hline
\end{tabular}

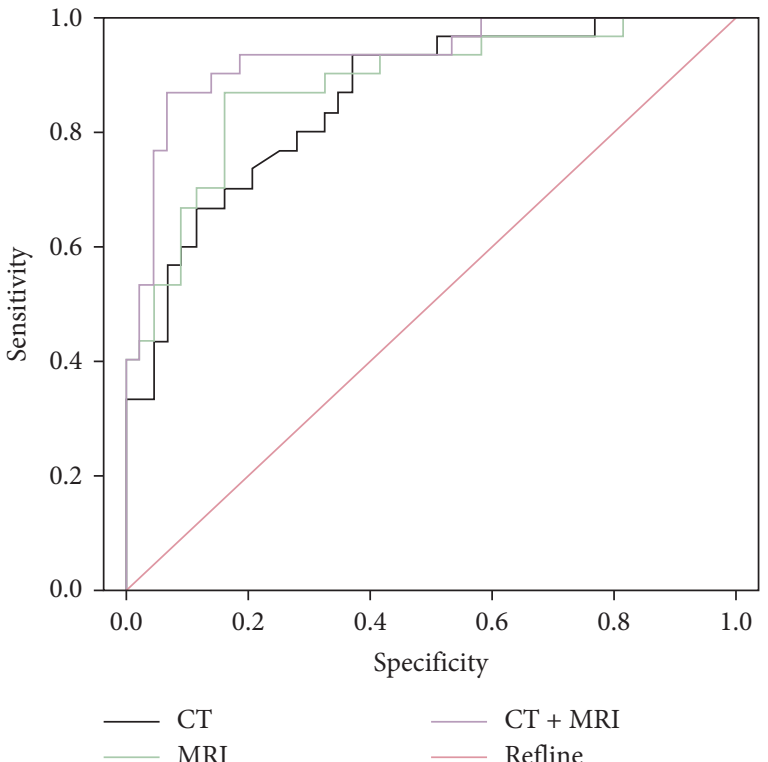

FIgUre 2: The diagnostic ROC curve of MRI combined with CT in patients with CCA.

higher detection rate in patients with CCA. Briefly, MRI combined with CT examination is helpful to the early diagnosis of CCA patients and can guide the clinical diagnosis and treatment.

It is well-known that fibrous tissue, mucin, coagulative necrosis, and malignant tumor tissue cells are the main components of the mass. And according to the area, tissue type, and distribution characteristics of the tumor, there are obvious differences between different components [19]. It has been found that the imaging manifestations of CCA mainly depend on the pathological type, the distribution of fibrous tissue, and tumor cells [20]. The tumor cells are distributed around the tumor, and the proliferating fibrous tissue is located in the center of the tumor. The contrast agent diffuses slowly in the fibrous tissue and can obtain clear images [21].

In this study, the CT group had more cases of delayed enhancement, peripheral bile duct dilation, and hepatic capsule depression than MRI. The number of uneven enhancement cases in the CT group was less than that of MRI. The above results indicate that MRI and CT have typical imaging characteristics in patients with CCA. The diagnosis of different lesions can be confirmed through imaging features. In order to further analyze the diagnostic value of MRI and CT in patients with CCA, ROC curves were drawn in this study. The results showed that the diagnostic sensitivity and specificity of MRI combined with CT in patients with CCA were higher than those of single MRI and CT. Therefore, MRI combined with CT examinations should be strengthened for patients with suspected CCA. However, the number of samples in our study is a little small. We will continue to collect data in the follow-up to better confirm our conclusions.

\section{Conclusion}

In conclusion, MRI combined with CT can achieve a high diagnostic coincidence rate in patients with CCA and has typical imaging features. Moreover, the sensitivity and specificity of the combined examination are high. Therefore, MRI combined with CT can provide imaging basis for the clinical diagnosis and treatment of CCA.

\section{Data Availability}

The datasets used and/or analyzed during the present study are available from the corresponding author on reasonable request.

\section{Conflicts of Interest}

The authors declare that they have no conflicts of interest.

\section{References}

[1] P. X. Huang, Q. L. Song, S. J. Di et al., "The diagnostic value of endoscopic balloon dilatation combined with biliary cytology in cholangiocarcinoma," Chinese Journal of Hepatobiliary Surgery, vol. 27, no. 3, pp. 202-205, 2021.

[2] Z. F. Wang, F. E. Hao, L. Zhu et al., "CT and MRI imaging features of pancreatic acinar cell carcinoma," Chinese Journal of Digestive Surgery, vol. 19, no. 5, pp. 552-558, 2020.

[3] Y.-H. Yang, Q. Deng, T.-B. Yang et al., "A case report of cholangiocarcinoma combined with moderately differentiated gastric adenocarcinoma," Medicine, vol. 98, no. 30, Article ID e16332, 2019.

[4] Y. Zhou, D. Li, L. Long et al., "Analysis of enhanced mode of mixed liver cancer and the diagnostic value of contrast-enhanced ultrasound combined with enhanced CT/MRI and tumor markers," Chinese Journal of Ultrasound Imaging, vol. 29, no. 9, pp. 754-760, 2020.

[5] Z. P. Qi, J. L. Li, X. L. Zhang et al., "The diagnostic value of serum tumor markers combined with multi-slice spiral CT and MRI in the diagnosis of cholangiocarcinoma and its relationship with tissue invasion molecules," Advances in Modern Biomedicine, vol. 19, no. 20, pp. 161-164, 2019.

[6] D. T. Jing and D. Li, "The diagnostic efficacy and imaging characteristics of CT combined with MRI in primary central nervous system lymphoma," Guizhou Medicine, vol. 43, no. 9, pp. 1469-1471, 2019. 
[7] H. Y. Heng, X. Ding, G. Q. Chen et al., "Analysis of CT and MRI imaging features of massive intrahepatic cholangiocarcinoma," Chongqing Medicine, vol. 49, no. 8, pp. 118-121, 2020.

[8] Biliary Surgery Group of the Chinese Medical Association Surgery Branch, PLA Committee of Hepatobiliary Surgery of the PLA, "Guidelines for the diagnosis and treatment of hilar cholangiocarcinoma (2013 edition)," Chinese Journal of Surgery, no. 10, pp. 865-871, 2013.

[9] Y. C. Fang, Q. Wang, Q. Tang et al., "Analysis of the diagnostic value of MSCT and MRI dynamic enhancement scan for high blood supply intrahepatic cholangiocarcinoma and hepatocellular carcinoma," Journal of Practical Hepatology, vol. 23, no. 6, pp. 131-134, 2020.

[10] S. H. Yang, Z. J. Liao, S. Hu et al., "Expression and significance of eukaryotic translation initiation factor-5A2 in intrahepatic cholangiocarcinoma," Journal of Kunming Medical University, vol. 42, no. 5, pp. 24-28, 2021.

[11] D. Xiao, T. Han, C. Zhai et al., "The value of MRI combined with MSCT in the diagnosis of preoperative Tand N staging of colorectal cancer," Chinese Journal of CT and MRI, vol. 18, no. 10, pp. 139-141, 2020.

[12] A. J. Donato, D. R. Machin, and L. A. Lesniewski, "Mechanisms of dysfunction in the aging vasculature and role in agerelated disease," Circulation Research, vol. 123, no. 7, pp. 825-848, 2018.

[13] H. Li, S. He, K. Fan et al., "Relationship between the onset date of the Meiyu and the South Asian anticyclone in April and the related mechanisms," Climate Dynamics, vol. 52, no. 1-2, pp. 209-226, 2019.

[14] T. Song, J. Y. Liu, and J. P. Lu, "Computed tomography and magnetic resonance imaging features analysis of pancreatic acinar cell carcinoma," Journal of Second Military Medical University, vol. 40, no. 3, pp. 110-114, 2019.

[15] I. Roy and R. H. Kripalani, "The role of natural factors (part 1): addressing on mechanism of different types of ENSO, related teleconnections and solar influence," Theoret Applied Climatol, vol. 137, no. 1-2, pp. 469-480, 2019.

[16] Y. P. Zeng, J. D. Luo, X. M. Leng et al., "CT and MRI differential diagnosis of hepatocellular carcinoma with cholangiocarcinoma thrombus and intrahepatic cholangiocarcinoma," Modern Oncology, vol. 28, no. 1, pp. 108-113, 2020.

[17] C. Q. Long, Z. Q. Zhou, W. J. He et al., “Application of multislice spiral CT multiplanar reconstruction technology in the diagnosis of cholangiocarcinoma," Chinese Journal of CT and MRI, vol. 18, no. 5, pp. 32-34, 2020.

[18] Y. Qi, J. Guo, X. H. Zhou, and X. B. Fan, "CT and MRI differential diagnosis of intrahepatic cholangiocarcinoma and atypical liver abscess," Journal of Medical Imaging, vol. 30, no. 8, pp. 103-106, 2020.

[19] J. Vlachogiannakos, T. Voulgaris, D. Karagiannakis et al., "Tu1586-do ppis affect the incidence of liver-related complications and overall survival in patients with cirrhosis? The experience of a Greek tertiary hospital," Gastroenterology, vol. 156, no. 6, 1369 pages, 2019.

[20] Q. J. Wu, M. L. Xiong, X. Y. Wang et al., "Clinical case analysis of dual primary carcinomas of simultaneous hepatocellular carcinoma and intrahepatic cholangiocarcinoma," West China Medicine, vol. 34, no. 3, pp. 94-99, 2019.

[21] B. S. Yang, M. Yuan, T. Y. Chen et al., "Predictive value of enhanced MRI texture analysis for the efficacy and prognosis of intrahepatic cholangiocarcinoma patients undergoing hepatic artery infusion chemotherapy," Journal of Interventional Radiology, vol. 29, no. 12, pp. 29-35, 2020. 